\title{
Expression and clinical implications of enhancer of Zeste homolog 2 and p53 protein in squamous cell carcinoma and precancerous lesions in the cervix
}

\author{
H.M. Zhang', S.Q. Chen ${ }^{2}$ and S.Z. Yao ${ }^{2}$ \\ ${ }^{1}$ GuiZhou Provincial People's Hospital, Guiyang, China \\ ${ }^{2}$ The First Affiliated Hospital, Sun Yat-Sen University, Guangzhou, China \\ Corresponding author: H.M. Zhang \\ E-mail: zhanghmvip@163.com \\ Genet. Mol. Res. 15 (2): gmr.15027408 \\ Received August 11, 2015 \\ Accepted January 8, 2016 \\ Published June 17, 2016 \\ DOI http://dx.doi.org/10.4238/gmr.15027408
}

\begin{abstract}
We investigated the expression and clinical implications of enhancer of Zeste homolog 2 (EZH2) and p53 protein in cervical squamous cell carcinoma (SCC) and precancerous lesions. EZH2 and p53 expressions in SCC (168), cervical intraepithelial neoplasia (CIN)-I (19), CIN-II (35), and normal tissues (30) were detected by streptavidin-peroxidase-conjugation. The correlation between coexpression of EZH2 and p53 protein and the clinic pathological features and prognosis of SCC were discussed. The positive expression rates of EZH2 and p53 were 6.7, 37.0, and 75.6\%, and 3.3, 21.1, and $39.3 \%$ in normal cervical tissues, CIN, and SCC, respectively, which were significantly different $(\mathrm{P}<0.05)$. The positive expression rate of EZH2 and p53 protein in SCC patients with and without lymph node metastasis was 82.9 and $70.4 \%$ (EZH2) and 45.7 and 34.7\% (p53), respectively, which was also a significant difference $(\mathrm{P}<0.05)$. The progression-free survival (PFS) rates in followed-up patients $(\mathrm{N}=143)$ who were EZH2- and p53-negative, EZH2- or p53-positive, and EZH2-
\end{abstract}


and p53-positive were $71.3 \pm 1.9,66.1 \pm 2.0$, and $51.3 \pm 3.8$ months, respectively, which was a significant difference $(\mathrm{P}<0.001)$; the overall survival among these groups was $72.9 \pm 1.1,68.6 \pm 1.8$, and $57.4 \pm$ 3.4 months, respectively $(\mathrm{P}<0.001)$. Multivariate analyses revealed that EZH2 expression, lymph node metastasis, and tumor staging were independent prognostic factors of SCC. EZH2 and p53, which affect lymph node metastasis and prognosis of SCC, may play a key role in the occurrence and development of SCC.

Key words: EZH2; p53 protein; Squamous cell carcinoma; Cervical intraepithelial neoplasia; Immunohistochemistry;

Survival analysis

\section{INTRODUCTION}

Cervical carcinoma, a high incidence cancer type, affects the female genitalia; squamous cervical carcinoma (SCC) is the most common tumor affecting the cervix. There has been a growing incidence of cervical carcinoma over the past few years, with a large percentage of cases affecting young women (Parkin et al., 2001). Cervical intraepithelial neoplasia (CIN) is a precancerous cervical lesion, and must be diagnosed and treated early to avoid cancer progression. Enhancer of zeste homolog 2 (EZH2) inhibits cell differentiation and target gene expression. EZH2, in conjunction with the p53 protein, induces tumor cell proliferation, metastasis, and immortalization. Highly expressed in various malignant tumors, $\mathrm{EZH} 2$ and $\mathrm{p} 53$ protein are closely related to the degree of malignancy and prognosis of tumors (Bracken et al., 2003; Tang et al., 2004; Dong et al., 2007). In this study, we attempted to detect the expression of EZH2 and p53 in different types of cervical lesions by immune histochemical analysis, and discuss their clinical implications.

\section{MATERIAL AND METHODS}

\section{Patients and specimen collection}

Cervical lesions were collected from 253 patients receiving surgical treatment for cervical cancer from January 2005 to December 2006 at the First Affiliated Hospital of Sun Yatsen University. Among these, 30 patients underwent a hysterectomy for hysteromyoma (normal control), and 19 and 35 patients were at stage II and III of CIN, respectively. One hundred and sixty-eight patient shad SCC. The cancer stages were determined according to the 2009 International Federation of Gynecology and Obstetrics (FIGO) staging system: 13, 71, and 84 cases were at stage IA, IB, and IIA of cancer. Among the 168 SCC patients, 98 showed lymph node metastasis, while the others did not. All cases were followed up for 60-84 months; all 25 patients whose follow-up was discontinued were women with SCC (lost follow-up rate: $9.9 \%$ ).

\section{Immunohistochemistry}

The expression of EZH2 and p53 was detected by the SP method, using citrate buffer $(\mathrm{pH}=6.0)$ for repair. The staining intensity was determined by comparing the sample with the 
positive and negative controls. The proportion of positive cells in each slide was calculated; samples with $<30$ and $\geq 30 \%$ positive cells were considered as negative and positive samples, respectively.

\section{Statistical analysis}

All statistical analyses were performed using SPSS v.18.0 (IBM, Armonk, NY, USA). The count data was analyzed by the $\chi^{2}$ test. The data was also subjected to KaplanMeier survival analysis and log-rank test. Multivariate analysis was performed using Cox's proportional hazard model, with significance set at $\alpha=0.05$.

\section{RESULTS}

\section{EZH2 and p53 expression in cervical lesions}

The EZH2 and p53 protein expression in different cervical lesions were analyzed by immunohistochemistry (Figure 1). The positive expression rate of EZH2 in normal cervical tissue, and CIN, and SCC tissues was 6.7\% (2/32), 37.0\% (20/54), and 75.6\% (127/168), respectively, while that of p53 in these tissues was $3.3 \%(1 / 30), 21.1 \%(11 / 54)$, and $39.3 \%$ (66/168), respectively; all differences were statistically significant $(\mathrm{P}<0.05)$. The positive expression rates of EZH2 and $\mathrm{p} 53$ in SCC patients $(\mathrm{N}=168)$ with $(\mathrm{N}=70)$ and without $(\mathrm{N}$ = 98) lymph node metastasis were $82.9 \%(58 / 70)$ and $70.4 \%(69 / 98)$ and $45.7 \%(32 / 70)$ and $34.7 \%$ (34/98), respectively; these differences were found to be statistically significant $(\mathrm{P}<$ $0.05)$. The patients were divided into 3 groups based on the expression of EZH2 and p53: EZH2- and p53-positive, EZH2- or p53-positive, and EZH2- and p53-negative. EZH2 and p53 expressions in different types of cervical lesions are summarized in Table 1.

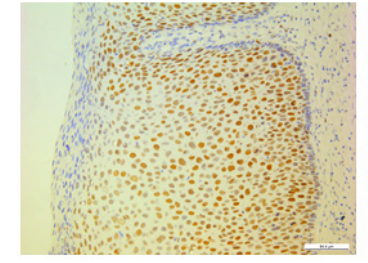

A

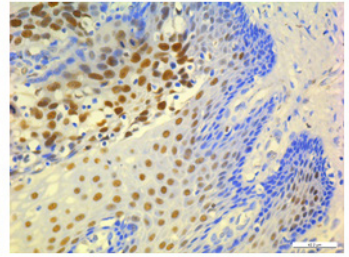

B

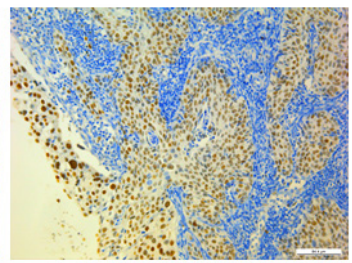

$\mathrm{C}$

Figure 1. EZH2 and p53 protein expression in CIN and SCC tissues. A. Positive expression of p53 in CINI (immunohistochemistry; 200X magnification). B. Positive expression of EZH2 at the boundary between CIN and SCC (immunohistochemistry; 400X magnification). C. Positive expression of EZH2 in SCC (immunohistochemistry; 200X magnification). All data expressed as percentages. $\mathrm{CIN}=$ cervical intraepithelial neoplasia; $\mathrm{EZH} 2=$ enhancer of Zeste homolog 2.

Table 1. Expression of the enhancer of Zeste homolog 2 (EZH2) and p53 protein in different types of cervical lesions.

\begin{tabular}{l|c|c|c|c|c}
\hline Group & Cases & Positive for EZH2 and p53 & Positive for EZH2 or p53 & Negative for EZH2 and p53 & P value \\
\hline Normal control & 30 & $1(3.3)$ & $1(3.3)$ & $28(93.3)$ & 0.001 \\
\hline CIN II & 19 & $2(10.5)$ & $6(31.6)$ & $11(57.9)$ & 0.009 \\
\hline CIN III & 35 & $4(11.4)$ & $14(40.0)$ & $17(48.6)$ & 0.039 \\
\hline SCC & 168 & $51(30.4)$ & $92(54.8)$ & $25(14.9)$ & 0.027 \\
\hline
\end{tabular}




\section{Correlation between EZH2 and p53 expression and clinic pathological features of SCC}

The correlation between EZH2 and p53 expression and the clinic pathological features of SCC are summarized in Table 2. EZH2 and p53 expression differed significantly between patients with difference stages of SCC (IB and IIA; P $<0.05$ ), and between patients with and without lymph node metastasis $(\mathrm{P}<0.05)$.

\begin{tabular}{|c|c|c|c|c|c|}
\hline Clinicopathological features & Cases & Positive for EZH2 and p53 $(\mathrm{N}=51)$ & Positive for EZH2 or $\mathrm{p} 53(\mathrm{~N}=92)$ & Negative for EZH2 and $\mathrm{p} 53(\mathrm{~N}=25)$ & \\
\hline \multicolumn{6}{|c|}{\begin{tabular}{l|l} 
Positive for EZH2 and p53 $(\mathrm{N}=51)$ & Positive for EZH2 or p53 $(\mathrm{N}=92)$ \\
\end{tabular}} \\
\hline Stage IA & 13 & 4 & 4 & 5 & 0.377 \\
\hline Stage IB & 71 & 16 & 42 & 13 & 0.021 \\
\hline Stage IIA & 84 & 31 & 46 & 7 & 0.019 \\
\hline \multicolumn{6}{|l|}{ Lymph node metastasis } \\
\hline No & 98 & 23 & 59 & 16 & 0.014 \\
\hline Yes & 70 & 28 & 33 & 9 & 0.027 \\
\hline \multicolumn{6}{|l|}{ Size of local foci $(\mathrm{cm})$} \\
\hline$<4$ & 137 & 41 & 78 & 18 & 0.077 \\
\hline$\geq 4$ & 31 & 10 & 14 & 7 & 0.311 \\
\hline \multicolumn{6}{|l|}{ Microinfiltration of vessels } \\
\hline No & 139 & 42 & 79 & 18 & 0.097 \\
\hline Yes & 29 & 9 & 13 & 7 & 0.379 \\
\hline
\end{tabular}

\section{Survival rate of SCC patients with varying EZH2 and p53 expression}

Progression-free survival (PFS) in followed-up SCC patients $(\mathrm{N}=143)$ who were EZH2- and p53-negative, EZH2- or p53-positive, and EZH2- and p53-positive was $71.3 \pm$ $1.9,66.1 \pm 2.0$, and $51.3 \pm 3.8$ months, respectively; the differences between the cases were statistically significant $(\mathrm{P}<0.001$; Figure 2$)$. EZH2- and p53-protein negative, EZH2- or p53positive, and EZH2- and p53-positive was $72.9 \pm 1.1,68.6 \pm 1.8$, and $57.4 \pm 3.4$ months, the differences between the cases were statistically significant $(\mathrm{P}<0.001$; Figure 3$)$.

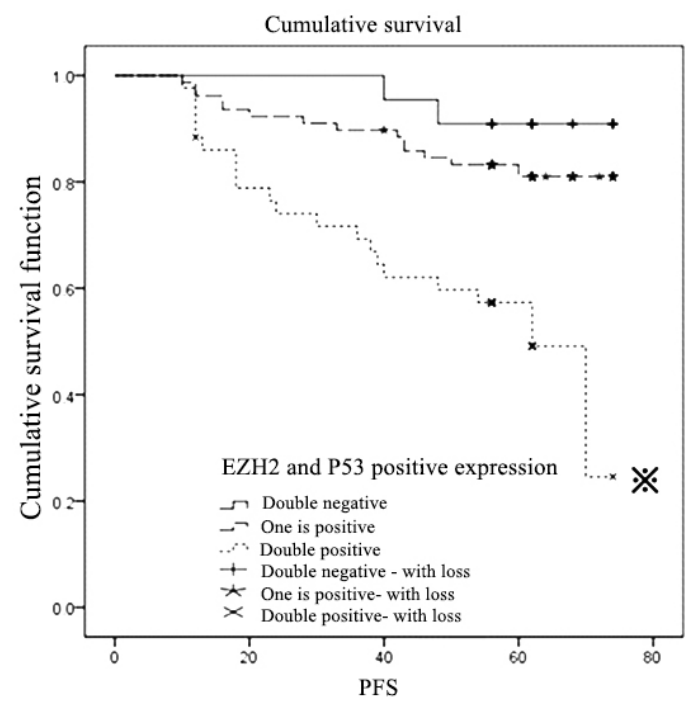

Figure 2. Progression-free survival (PFS) curve of SCC with varying EZH2 and p53 expression. SCC = squamous cell carcinoma; EZH2 = enhancer of Zeste homolog 2. 


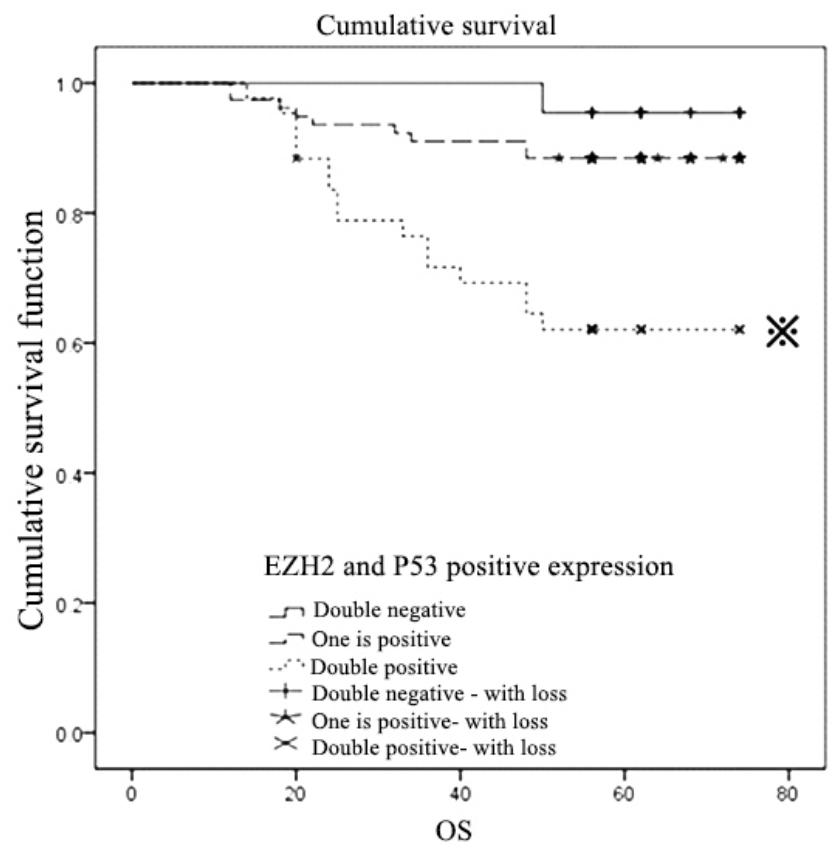

Figure 3. Survival curve of SCC cases with differential EZH2 and p53 expression. SCC: squamous cell carcinoma; EZH2: enhancer of Zeste homolog 2.

\section{Univariate and multivariate analysis of factors influencing the prognosis of SCC}

Univariate analysis revealed a correlation between the age and body mass index (BMI) of patients, and the EZH2 and p53 expression and SCC prognosis (Table 3). The multivariate analysis indicated that the EZH2 expression, lymph node metastasis, and tumor staging were independent factors influencing the prognosis of SCC (Table 4).

Table 3. Univariate analysis of factors influencing the prognosis of 143 squamous cell carcinoma (SCC) cases.

\begin{tabular}{|c|c|c|c|c|}
\hline Factor & & Cases & Survival time (months, means \pm SD) & P value \\
\hline \multirow[t]{2}{*}{ Age (years) } & $<50$ & 91 & $68.9 \pm 1.2$ & 0.032 \\
\hline & $\geq 50$ & 52 & $63.7 \pm 0.9$ & \\
\hline \multirow[t]{2}{*}{ Occupation } & Farmer & 35 & $65.7 \pm 1.2$ & 0.721 \\
\hline & Others & 108 & $65.9 \pm 1.7$ & \\
\hline \multirow[t]{2}{*}{ Familial history } & No & 126 & $66.7 \pm 1.8$ & 0.498 \\
\hline & Yes & 17 & $64.2 \pm 0.7$ & \\
\hline \multirow[t]{2}{*}{ Childbearing history } & Having borne no children & 2 & $61.3 \pm 0.9$ & 0.278 \\
\hline & Having borne children & 141 & $65.9 \pm 1.5$ & \\
\hline \multirow[t]{2}{*}{ Menopause } & No & 87 & $68.3 \pm 1.7$ & 0.375 \\
\hline & Yes & 56 & $64.1 \pm 2.0$ & \\
\hline \multirow{2}{*}{ BMI $\left(\mathrm{kg} / \mathrm{m}^{2}\right)$} & $<24$ & 96 & $70.1 \pm 0.9$ & 0.023 \\
\hline & $\geq 24$ & 47 & $64.9 \pm 1.2$ & \\
\hline \multirow[t]{2}{*}{ EZH2 } & - & 35 & $73.3 \pm 0.7$ & $<0.001$ \\
\hline & + & 108 & $63.5 \pm 1.9$ & \\
\hline \multirow{2}{*}{ p53 } & - & 87 & $68.9 \pm 1.6$ & \multirow[t]{2}{*}{0.018} \\
\hline & + & 56 & $61.3 \pm 2.8$ & \\
\hline
\end{tabular}

EZH2: enhancer of Zeste homolog 2; BMI: body mass index. 
Table 4. Multivariate analysis of prognostic factors of squamous cell carcinoma (SCC; $N=143$ ).

\begin{tabular}{l|c|c|c|c|c}
\hline Factor & B value & Standard error & Wald value & RR (95\%CI) & P value \\
\hline EZH2 expression & 2.152 & 1.025 & 4.409 & $1.735(1.056-2.379)$ & 0.036 \\
\hline p53 expression & 0.574 & 0.421 & 1.859 & $1.147(0.403-1.585)$ & 0.173 \\
\hline Lymph node metastasis & 1.999 & 0.629 & 10.110 & $2.773(1.364-3.631)$ & 0.001 \\
\hline Tumor stage & 1.414 & 0.505 & 7.833 & $2.127(1.351-2.794)$ & 0.005 \\
\hline Age & -0.015 & 0.019 & 0.561 & $0.993(0.477-1.021)$ & 0.454 \\
\hline BMI & -0.082 & 0.061 & 1.825 & $0.985(0.955-1.005)$ & 0.177 \\
\hline
\end{tabular}

$\mathrm{SCC}=$ squamous cell carcinoma $; \mathrm{EZH} 2=$ enhancer of Zeste homolog $2 ; \mathrm{BMI}=$ body mass index.

\section{DISCUSSION}

Two major types of proteins with conservative structure and functions are responsible for regulating the cell proliferation and differentiation post-embryonic development: thepolycomb (PcG) and trithorax (TrxG) groups (Mills, 2010). EZH2 is a PcG protein that promotes cell proliferation, inhibits cell differentiation and target gene expression, and is closely associated with tumors. The EZH2 gene was first identified in humans in 1996 (Hobert et al., 1996), and was found to be homologous to the EZH2 gene in fruit flies; moreover, the resultant protein also possessed similar structure and functions to that seen in fruit flies. EZH2 is localized to chromosome 7q35, regulates cell growth, and promotes cell proliferation by inhibiting the target genes in the chromatin. EZH2 inhibits transcription, and thus reduces the activity of various genes, especially the expression of factors inhibiting tumor metastasis, such as TIMP (Shin and Kim, 2012) and RKIPz (Ren et al., 2012). EZH2 generally promotes tumor cell invasion and metastasis and reduces the expression of target genes by inhibiting the binding of transcription factors to DNA. It acts on the $\mathrm{pRb} 2 / \mathrm{p} 130 \mathrm{C}$-terminal domain that is associated with histone deacetylase (HDAC1). By restoring the activity of the cyclin A promoter, mediated by the pRb2/p130-HDAC1 complex, EZH2 accelerates the cell cycle progression and malignant transformation (Tonini et al., 2004).

EZH2 is expressed in tumors of the urinary system, breast tumors, and lymphoma; the expression of EZH2 in middle- and late-stage tumors and metastatic tumors is much higher than that in early-stage tumors and non-metastatic tumors. This implies that EZH2 expression is related to tumor invasion and metastasis (Visser et al., 2001; Hoffmann et al., 2007; Kunju et al., 2011). Bachmann et al. (2006) and Zhang et al. (2006) also demonstrated that EZH2 promoted the occurrence, development, and invasion of endometrial carcinoma. In this study, normal cervical squamous epithelia, CIN II, CIN III, and SCC tissues all showed increased expression of EZH2; moreover, the latter was also expressed in precancerous lesions. This suggested the role of the activation and expression of EZH2 in the onset and development of SCC. The prevalence of $E Z H 2$ was greater in patients with lymph node metastasis; therefore, EZH2 expression is an adverse factor that promotes tumor progression.

However, some studies have also shown that EZH2 is not a direct factor inducing tumor occurrence and development. EZH2 alters cell proliferation and differentiation by regulating the expression of other genes or proteins, which in turn leads to malignancy. The factors regulated by EZH2 include matrix metalloproteinase $(M M P)$, heat shock protein (HSP), and metastasis-associated gene (MTA). p53 protein expression is related to cervical HPV infection and the occurrence of cervical carcinoma (Zhang et al., 2006). However, the interactions between EZH2 and p53 in cervical carcinoma remainnunclear. Recent reports have suggested a negative correlation between EZH2 and p53 expression; moreover, wild-type 
p53 is believed to bind to EZH2 promoter, thereby inhibiting EZH2 expression, and arresting human fibroblasts at the $\mathrm{G}_{2}$ phase, inhibiting its proliferation, and inducing apoptosis (Tang et al., 2004). Bracken et al. (2003) reported conflicting results in osteosarcoma cells. In these cells, silencing of the $E Z H 2$ gene by RNA interference led to downregulation of the $p 53$ gene. Dong et al. (2007) demonstrateda positive correlation between EZH2 expression and that of mutant-type 533 protein in breast cancer. It was inferred that the two proteins acted in synergy during the development of breast cancer, although the specific mechanism was not elucidated.

In this study, we observed a correlation between EZH2 and p53 expression and lymph node metastasis and tumor staging in SCC. Simultaneous detection of EZH2 and p53 helped predict the prognosis of SCC, which was consistent with the previous discovery that EZH2 and p53 expression is associated with an increase in tumor metastasis. Kaplan-Meier survival analysis indicated that the PFS and OS of EZH2- and p53-positive SCC patients were significantly lower than those of EZH2- and p53-negative, or EZH2- or p53-positive patients. Multivariate analyses revealed that lymph node metastasis, tumor stage, and the EZH2 expression were independent prognostic factors of SCC. Hence, the study into the mechanism of action of EZH2 and p53 protein in cervical carcinoma contributes to the understanding of the molecular biology of tumor occurrence and development, and helps predict the malignant transformation of cervical lesions. Similar to factors such as infiltration of deep cervical muscles, having multiple sexual partners, and complicating pregnancy (Zhang et al., 2004), EZH2 expression plays a key role in the diagnosis, treatment, and prognostic evaluation of cervical carcinoma and precancerous lesions.

In conclusion, EZH2 and p53 are involved in the development of SCC, independently or in synergy. The detection of EZH2 and p53 expression can benefit the prediction of tumor stage, lymph node metastasis, and prognosis of SCC compared to the detection of EZH2 or p53 alone.

\section{Conflicts of interest}

The authors declare no conflict of interest.

\section{ACKNOWLEDGMENTS}

Research supported by the Social Development Project of Guizhou province, China [\#(2014) 7015].

\section{REFERENCES}

Bachmann IM, Halvorsen OJ, Collett K, Stefansson IM, et al. (2006). EZH2 expression is associated with high proliferation rate and aggressive tumor subgroups in cutaneous melanoma and cancers of the endometrium, prostate, and breast. J. Clin. Oncol. 24: 268-273. http://dx.doi.org/10.1200/JCO.2005.01.5180

Bracken AP, Pasini D, Capra M, Prosperini E, et al. (2003). EZH2 is downstream of the pRB-E2F pathway, essential for proliferation and amplified in cancer. EMBO J. 22: 5323-5335. http://dx.doi.org/10.1093/emboj/cdg542

Dong GL, Zhang XL and Yuan WQ (2007). EZH2 and p53 expression in breast cancer and correlation. Zhong Guo Zhong Liu Lin Chuang 34: 931-933.

Hobert O, Jallal B and Ullrich A (1996). Interaction of Vav with ENX-1, a putative transcriptional regulator of homeobox gene expression. Mol. Cell. Biol. 16: 3066-3073. http://dx.doi.org/10.1128/MCB.16.6.3066

Hoffmann MJ, Engers R, Florl AR, Otte AP, et al. (2007). Expression changes in EZH2, but not in BMI-1, SIRT1, DNMT1 or DNMT3B are associated with DNA methylation changes in prostate cancer. Cancer Biol. Ther. 6: 1403- 
1412. http://dx.doi.org/10.4161/cbt.6.9.4542

Kunju LP, Cookingham C, Toy KA, Chen W, et al. (2011). EZH2 and ALDH-1 mark breast epithelium at risk for breast cancer development. Mod. Pathol. 24: 786-793. http://dx.doi.org/10.1038/modpathol.2011.8

Mills AA (2010). Throwing the cancer switch: reciprocal roles of polycomb and trithorax proteins. Nat. Rev. Cancer 10: 669-682. http://dx.doi.org/10.1038/nrc2931

Parkin DM, Bray F, Ferlay J and Pisani P (2001). Estimating the world cancer burden: Globocan 2000. Int. J. Cancer 94: 153-156. http://dx.doi.org/10.1002/ijc. 1440

Ren G, Baritaki S, Marathe H, Feng J, et al. (2012). Polycomb protein EZH2 regulates tumor invasion via the transcriptional repression of the metastasis suppressor RKIP in breast and prostate cancer. Cancer Res. 72: 3091-3104. http://dx.doi. org/10.1158/0008-5472.CAN-11-3546

Shin YJ and Kim JH (2012). The role of EZH2 in the regulation of the activity of matrix metalloproteinases in prostate cancer cells. PLoS One 7: e30393. http://dx.doi.org/10.1371/journal.pone.0030393

Tang X, Milyavsky M, Shats I, Erez N, et al. (2004). Activated p53 suppresses the histone methyltransferase EZH2 gene. Oncogene 23: 5759-5769. http://dx.doi.org/10.1038/sj.onc.1207706

Tonini T, Bagella L, D'Andrilli G, Claudio PP, et al. (2004). Ezh2 reduces the ability of HDAC1-dependent pRb2/p130 transcriptional repression of cyclin A. Oncogene 23: 4930-4937. http://dx.doi.org/10.1038/sj.onc.1207608

Visser HP, Gunster MJ, Kluin-Nelemans HC, Manders EM, et al. (2001). The Polycomb group protein EZH2 is upregulated in proliferating, cultured human mantle cell lymphoma. Br. J. Haematol. 112: 950-958. http://dx.doi. org/10.1046/j.1365-2141.2001.02641.x

Zhang WH, Wu LY, Ba P and Wang F (2004). Istage B and IIA cervical cancer the prognosis of patients with factor analysis. Chin. J. Oncol 26: 490-492.

Zhang WM, Shuai CX, Zheng FY, Huang YP, et al. (2006). Fragile histidine triplet gene expression in cervical lesions and cervical cancer before and its relationship with 553 and HPV16/18. Chin. J. Oncol 28: 452-455. 